\title{
Itinerarios de cuerpos menstruantes: Descolonizando el cuerpo de la mujer*
}

\author{
Itineraries of menstruating bodies: descolonization the body of the woman
}

\author{
Itinerários de corpos menstruantes: descolonizar o corpo de mulheres
}

\begin{abstract}
Alejandra Paz Corvalán Navia**
Universidad de Playa Ancha, Chile. Facultad de Ciencias Sociales, Departamento de Psicología. Correo electrónico: alejandra.corvalan@upla.cl

Ariadna Maestre Gutiérrez***

Universidad del País Vasco, España. Correo electrónico: amaestgu@gmail.com

Revista Corpo-grafías: Estudios críticos de y desde los cuerpos / Volumen 4 - Número 4 / Enero - diciembre de 2017 / ISSN impreso 2390-0288, ISSN digital 2590-9398 / Bogotá, D.C., Colombia / pp. 77-91.

Fecha de recepción: 15 de septiembre de 2016

Fecha de aceptación: 7 de noviembre de 2016

Doi: https://doi.org/10.14483/25909398.13663

Cómo citar este artículo: Corvalán, A. Maestre, A. (2017, enero-diciembre). Itinerarios de cuerpos menstruantes: descolonizando el cuerpo de la mujer. Revista Corpo-grafías: Estudios críticos de y desde los cuerpos, 4(4), pp. 77-91 / ISSN 2390-0288.

*Artículo de investigación: El presente artículo es una reflexión independiente, construido desde el activismo feminista, la investigación cualitativa y el análisis teórico conjunto de las autoras. Este ensayo, conjunto a la investigación cualitativa, se llevó a cabo desde enero 2016 hasta abril del 2017.

** Licenciada y psicóloga por la Universidad Valparaíso, magíster en Análisis y Conducción de Grupos por la Universidad de Barcelona. Ha desarrollado su experiencia en trabajo con niños, niñas, jóvenes y mujeres en intervenciones en ámbitos terapéuticos y socioeducativos en contextos de exclusión y vulnerabilidad social. En la actualidad se desempeña como docente en el Departamento de Psicología de la Universidad de Playa Ancha y es encargada de Habilidades Transversales del Programa PACE MINEDUC, perteneciente a la Universidad Federico Santa María, donde trabaja con cultura juvenil y educación. En ambos espacios laborales se encuentra a cargo del eje transversal de género y feminismos.

*** Licenciada en Historia del Arte por la Universidad de La Laguna y la Universidad de Barcelona, España, posgrado en Antropología Social y Visual y máster en Antropología y Etnografía por la Universidad de Barcelona. En el 2014 cursó una estancia como investigadora invitada en la Universidad Autónoma Metropolita de México, en el departamento de Antropología Cultural. Ha trabajado como antropóloga en la Universidad de Barcelona y en las Islas Canarias. En la actualidad cursa el máster de Estudios Feministas y de Género de la Universidad del País Vasco y se desempeña como investigadora freelance en temas de género y feminismo
\end{abstract}

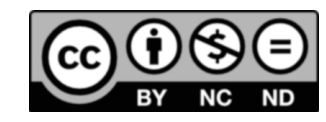




\section{Resumen}

El siguiente estudio da cuenta de diferentes testimonios de mujeres y la relación con su cuerpo y su menstruación. Testimonios que se convierten en herramienta de análisis respecto a la dominación simbólica que sufren estos cuerpos menstruantes. Mujeres de distintos territorios geográficos, con historias corporales donde el denominador común es la experiencia subjetiva de mecanismos de dominación encarnados.

Un estudio empírico que se guía por una etnografía, posteriormente complementada con itinerarios corporales que se constituyen como reflexiones escritas de las informantes, donde narran su historia corporal tomando la menstruación como eje principal.

Este artículo implica visibilizar en el espacio público aquello que ha estado relegado al tabú, a lo privado. Los cuerpos menstruantes y su configuración desde la dominación, evidencia cómo las mujeres han sido sistemáticamente coaccionadas para ser funcionales al sistema capitalista, sexista, clasista y patriarcal; y permite revelar cómo desde las prácticas cotidianas se rompen estos estereotipos.

Palabras clave: cuerpos menstruantes, feminismos, itinerarios corporales, menstruación, mujer, transnacionalidad.

\section{Abstract}

This research explain different testimonies of women and the relationship with their body and their menstruation. Testimonies that become a tool of analysis regarding the symbolic domination that these menstruating bodies suffer. Women of different geographic territories, with corporal histories where the common denominator is the subjective experience of mechanisms of domination.

An empirical study that is guided by an ethnography, later complemented with corporal itineraries that are constituted as written reflections of the informants where they narrate their corporal history taking the menstruation like main axis.

This article implies making visible in the public space that has been relegated to the taboo, to the private. The menstruating bodies and their configuration from domination, show how women have been systematically coerced to be functional to the capitalist, sexist, class and patriarchal system; and thus revealing how these stereotypes break from everyday practices. Keywords: menstruating bodies, feminisms, corporal itineraries, menstruation, woman, transnationality.

\section{Resumo}

O seguinte estudo dá a conhecer diferentes testemunhos de mulheres e da relação que têm com o seu corpo e a sua menstruação. Estes testemunhos convertem-se em ferramentas de análise relativamente à dominação simbólica que sofrem estes corpos menstruantes. Mulheres de distintos territórios geográficos, com histórias corporais em que o denominador comum é a experiência subjetiva de mecanismos de dominação encarnados.

Um estudo empírico que se guia por uma etnografia, posteriormente complementada com itinerários corporais que se constituem como reflexões escritas das informantes que narram a sua história corporal tomando a menstruação como eixo principal.

Este artigo implica dar uma maior visibilidade no espaço público ao que tem sido considerado tabu y da esfera privada. Os corpos menstruantes, e a sua configuração a partir da dominação, mostram como as mulheres foram sistematicamente coagidas para serem funcionais ao sistema capitalista, sexista, classicista e patriarcal, e revelam como a partir de práticas cotidianas se rompem esses estereótipos.

Palavras-chave: corpos menstruantes, feminismos, itinerários corpo, menstruação, mulher, transnacionalismo. 


\section{Introducción}

La cultura de la ocultación que rodea la menstruación implica la inexistencia de un foro público donde debatir esta «verdad» relativa a la experiencia femenina. El silencio resulta significativo a varios niveles. Primero, evita que la industria de protección sanitaria debe enfrentarse a una adecuada regulación. Segundo, alimenta la confusión que las chicas sienten al enfrentarse a lo que implica ser mujer. (...) En ausencia del verdadero debate, la realidad de la menstruación aparece irremediablemente sumida en las profundidades del mito.

Karen Houppert (2000, p. 252)

La menstruación y el ciclo menstrual son procesos cotidianos y normales que tiene lugar en el cuerpo de algunas mujeres con una periodicidad aproximada de veintiocho días durante cuarenta o cuarenta y cinco años de la vida, doce veces al año. Pese a lo anterior, la menstruación es uno de los grandes tabúes sociales e históricos existente sobre el cuerpo de la mujer. Este fenómeno se ha visto relegado al espacio privado y se ha construido un mito donde cualidades como impuro, sucio y enfermedad han permeado su significación. El sangrado natural adquiere valor principalmente por ser el productor del óvulo que puede ser fecundado, y siguiendo la misma lógica, es visto como un proceso mecánico para la reproducción y no como un proceso natural con el que las mujeres conviven en la cotidianidad de su vivencia corporal, generando una desconexión de la mujer con su cuerpo, y su menstruación.

La identidad de las mujeres se ha forjado históricamente a partir de diferentes narrativas teóricas que han sido promovidas por dispositivos: históricos, ideológicos, políticos, sociales y culturales. Temas vinculados a la salud mental y física de la mujer no han sido la excepción, tal cual como refiere Salvia:

(...) características de determinadas fases de la sexualidad femenina son interpretadas como una alteración de lo deseable y no como cualidades saludables y positivas, lo que ocurre en relación, por ejemplo, a la fase premenstrual, la fase menstrual, el puerperio o la menopausia (2012, p.16).

Esta producción simbólica respecto al cuerpo y la salud de las mujeres es solidaria con aquellos espacios donde se perpetúan desigualdades respecto al género.

Los tabúes menstruales en las sociedades occidentales, o con influencia de ellas, están construidos desde un imaginario androcéntrico, apoyado desde la misoginia tradicional de la historia occidental donde el cuerpo de la mujer se relaciona con lo monstruoso, con lo otro, y donde se refuerza la idea masculina que sangrar es algo maligno, ya que se relaciona con la enfermedad y con la muerte. 
La doctora Carme Valls con relación a este tema menciona:

Tanto la investigación médica, como la docencia y la asistencia sanitaria ha mirado a las mujeres "como si fuesen hombres» (...) la salud de las mujeres se ha estudiado y valorado sólo como salud reproductiva, y este enfoque ha impedido abordar la salud de las mujeres desde una perspectiva integral (2006, p. 72).

Esta cosmovisión masculina del sangrado y la corporalidad tiene como consecuencia la construcción del imaginario de la alteridad supeditado al canon hegemónico que promueve y universaliza un modelo «ideal» de mujer, manteniendo la subordinación del género en la construcción subjetiva, en la vinculación que se tiene con el cuerpo y a través de perpetuar relaciones donde se protege al hombre del contacto con la mujer durante la ocurrencia de este proceso (Corvalán, 2013) ${ }^{1}$.

1. Los tabúes menstruales se dan prácticamente en la mayoría de las culturas, bien por procesos de aculturación a través del colonialismo o porque ya tenían culturalmente admitido el estigma hacia la menstruación. En todo caso en los territorios donde hemos investigado, en la actualidad, existe culturalmente un estigma hacia la menstruación.

Fotografía por: Hernando Toro

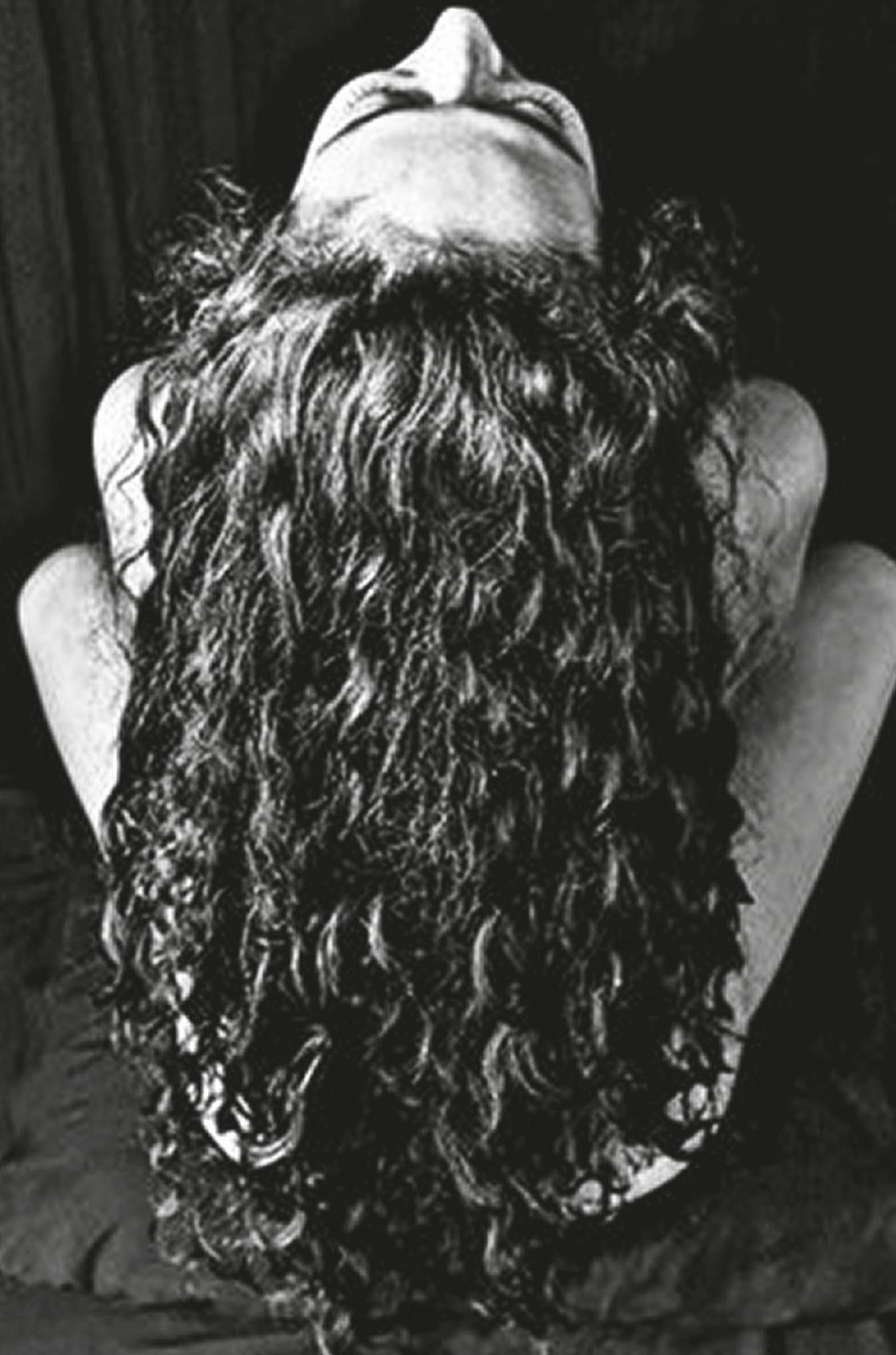


Esta producción simbólica en torno a la menstruación se ha perpetuado en la llamada "sociedad moderna», entendiendo la modernidad como una categoría colonial (Mignolo, 2007). Si bien Mignolo usa la categoría para hablar del colonialismo hacia todos los cuerpos colonizados, nosotras en este caso nos referimos hacia el cuerpo de la mujer como cuerpo colonizado durante la modernidad en Europa y en las zonas de expansión europea, a través del colonialismo, donde se implantó la construcción moderna del hombre blanco como ser universal y representante de la ciudadanía y donde el cuerpo de la mujer quedó excluido y separado de ese ser universal masculino europeo (Perona, 1995).

Ese cuerpo, no masculino, encontró en la modernidad y más tarde en los sistemas de salud hegemónicos su construcción actual en el cual el cuerpo de la mujer es el cuerpo de la madre y por tanto su cuerpo tiene un valor reproductivo, más tarde se convierte en un bien de consumo y con ello es coherente con una conceptualización que permite colonizar los cuerpos encauzándolos en el correcto orden del mundo desde una visión colonial y mercantilista; manteniendo de esta manera el sello de poder y control sobre la mujer, su cuerpo y su menstruación. Berbel refiriéndose a este mismo tópico explica que es de esta forma como las sociedades humanas han ido construyendo en torno a los procesos biológicos y fisiológicos de las mujeres un completo entramado ideológico con el fin de preservar como tabú todos los temas relacionados con el sexo femenino y, al mismo tiempo, mantener la situación de inferioridad «natural» de las mujeres en la esfera social (2001, p. 60).

Un rol fundamental to han cumplido los sistemas de salud hegemónicos implantados en el desarrollo de la modernidad; los cuales han permitido la normativización y reglamentación de los cuerpos, lo que interviene en cómo se configuran las relaciones de género promoviendo y universalizando un modelo «ideal» de mujer; lo cual mantiene la subordinación de las mujeres en los ámbitos, tanto profesionales como personales (Jiménez, 2008). Aproximadamente hasta mediados del siglo XX la ginecología estaba enfocada en el cuerpo de la mujer como cuerpo reproductivo, y es a partir de los años cincuenta del mismo siglo cuando se incorporan los estudios sobre la menstruación y el ciclo menstrual con el objetivo de desarrollar medicamentos anticonceptivos y así controlar, tanto el ámbito de la natalidad como de la reproducción de las mujeres que empiezan a incorporarse al mercado laboral de manera oficial, ergo se mantiene el dominio y los tabúes que se habían producido anteriormente para el cuerpo de la mujer a través de los sistemas de salud públicos o estatales (Fabiónavá, 2009; Valls, 2009; Wolf, 2013), obviando en estos espacios aspectos fisiológicos y experienciales que vive la mujer durante estos ciclos y en consecuencia no permitiendo que se naturalice como un proceso normal y cotidiano del cuerpo de las mujeres.

Es importante para nosotras destacar que pese a que hacemos una relación entre los cuerpos menstruantes y las mujeres, somos conscientes que no todas las mujeres tienen cuerpos menstruantes. Algunas no están representadas 
en este trabajo como pueden ser las transexuales, transgénero o aquellas que su aparato reproductor ha dejado de producir óvulos, por alguna presencia o ausencia de algún elemento específico o por haber alcanzado el climaterio. Con todo ello es innegable que la menstruación dentro de la cultura popular está asociada al cuerpo de la mujer y a su posibilidad de concebir (Corvalán, 2013).

\section{Etnografiando a mujeres menstruantes: del activismo a la investigación}

La etnografía, la investigación pueden (deben) servir para ser más conscientes de nosotras mismas, como humanas y como investigadoras, pero también para tomar distancias, no solo de nosotras, sino de eso que llamamos nuestra cultura. Pero todo eso no es posible sin un ejercicio de extrañamiento, sin desestabilizar la mirada, sin convertirnos en forasteras, en videntes.

Mari Luz Esteban (2011, p. 27).

Antes de explicar el abordaje metodológico, es importante resaltar que como investigadoras este estudio y su consiguiente artículo nos incluye a nosotras como parte de este experimento etnográfico. Nosotras pensamos y actuamos como sujetas activas en torno a re-significar (nuestro) el cuerpo de mujer. Es justamente en esta búsqueda de re-pensar el cuerpo en la que consideramos que la etnografía es la metodología que nos entrega mayores elementos para reflexionar desde las diferencias socioculturales entre cuerpos y hacer el ejercicio comprensivo de acercarnos a la vivencia y al significado de la menstruación, sin dejar de lado otras categorías sociales que marcan desigualdades y que jerarquizan relaciones entre seres sociales (Lugones, 2008). Es por eso que esta investigación en inicio se guió por una etnografía basada en entrevistas y conversaciones informales con diferentes mujeres donde explicaban su relación con la menstruación. En un segundo momento se optó por lo que nosotras denominamos itinerarios corporales de menstruación, tomando la idea de los itinerarios corporales de Mari Luz Esteban (2004, 2008), en los cuales se solicitaba a diferentes mujeres que construyeran un itinerario de vida de cómo es su vida como mujer menstruante y qué se entreteje en reflexiones escritas por parte de las propias informantes, dónde explican su historia corporal tomando la menstruación como eje de este itinerario. Estos relatos nos permitieron acceder no solo a las vivencias individuales de cada una, sino también a las relaciones que establecen con su cuerpo y a los contextos sociales en donde viven dichos cuerpos (Guilloó, 2013). A partir de la lectura de los itinerarios corporales menstruantes se nos permite construir la realidad social desde las voces de las mujeres menstruantes y las subjetividades que afloran en relación con su ciclo menstrual, su corporalidad, su género, entre otras. 
La investigación se ha llevado a cabo entre diferentes mujeres de Chile (Región de Valparaíso y Región Metropolitana) y España (Cataluña e Islas Canarias) ${ }^{2}$ rompiendo con los límites territoriales que se le acusa a la etnografía e incluyendo a mujeres de distintos ámbitos socioeconómicos y de diferentes territorios geopolíticos. El principal motivo que ha llevado a estos territorios tan diversos está relacionado con nuestra biografía personal, y no guiada por una elección académica. No obstante, se vuelve coherente con el emergente de esta propuesta que busca complejizar, pluralizar y particularizar el significado del concepto mujer.

Es importante destacar que este artículo no parte de una investigación académica al uso, sino de dos mujeres con un claro activismo hacia re-pensar su propio cuerpo. Nosotras con la participación en conversatorios, talleres, etc. hemos solicitado a amigas, conocidas y activistas que participen en los itinerarios menstruantes y a partir de eso, se ha reflexionado sobre el significado de la menstruación para las mujeres que están a nuestro alrededor. Resultado del proceso anterior, es que cada investigadora ha realizado el trabajo de campo en diferentes territorios de ambos países, primero en Cataluña donde ambas vivimos en un pasado cercano y donde las dos nos conocimos y nos acercamos hacia re-pensar nuestro cuerpo y la menstruación de la mano de movimientos feministas asociados al cuerpo y su gestión. Más tarde se hizo el mismo trabajo, la autora Alejandra Corvalán Navia en Chile y la autora Ariadna Maestre Gutiérrez en las Islas Canarias (España); territorios de origen a los cuales retornamos posterior a nuestra residencia en Cataluña.

Decidimos trabajar y reflexionar sobre este aprendizaje de lo que significa la menstruación en nuestro cuerpo y el tabú que existe hacia ella en nuestros círculos cercanos y no tan cercanos, a través de conversatorios en centros sociales o ponencias en distintas universidades.

En nuestro trabajo se marca una cierta diferencia entre los itinerarios menstruantes de mujeres en Cataluña con el resto, dado que en Cataluña las mujeres sí reflejan una mayor crítica hacia el tabú menstruante y cómo los sistemas de salud dan lectura al fenómeno. A diferencia de los otros territorios en los cuales los relatos de las mujeres menstruantes promueven construir otra cosmovisión del cuerpo de la mujer, la salud y la menstruación ${ }^{3}$. De todas formas todos los itinerarios nos muestran que la opresión hacia la mujer y su cuerpo es una problemática transnacional, dado la presencia de cierta similitud en sus itinerarios, no obstante, las diferencias presentes en el discurso de las informantes habla de cómo los actores y las instituciones occidentales han ayudado a construir y a mantener las barreras entre la cultura occidental y otras culturas diferentes (Jiménez, 2008), como se evidencia en los casos de las informantes de Chile e Islas Canarias.

\footnotetext{
2. La Región de Valparaíso y la Región Metropolitana están en la parte central de Chile, en esta última se encuentra la capital, Santiago de Chile. Cataluña está al noreste de la Península Ibérica, en la zona mediterránea, y las Islas Canarias se ubican al noroeste del continente africano ambas, pertenecen al Estado español.

3. Nos parece importante destacar, que cuando hablamos de Chile y España, no hablamos de nacionalidades, sino de territorios geopolíticos en los que habitan estos cuerpos menstruantes, dado que en esta investigación han participado mujeres con diferentes nacionalidades que no necesariamente corresponden con la nacionalidad del lugar donde residen.
} 


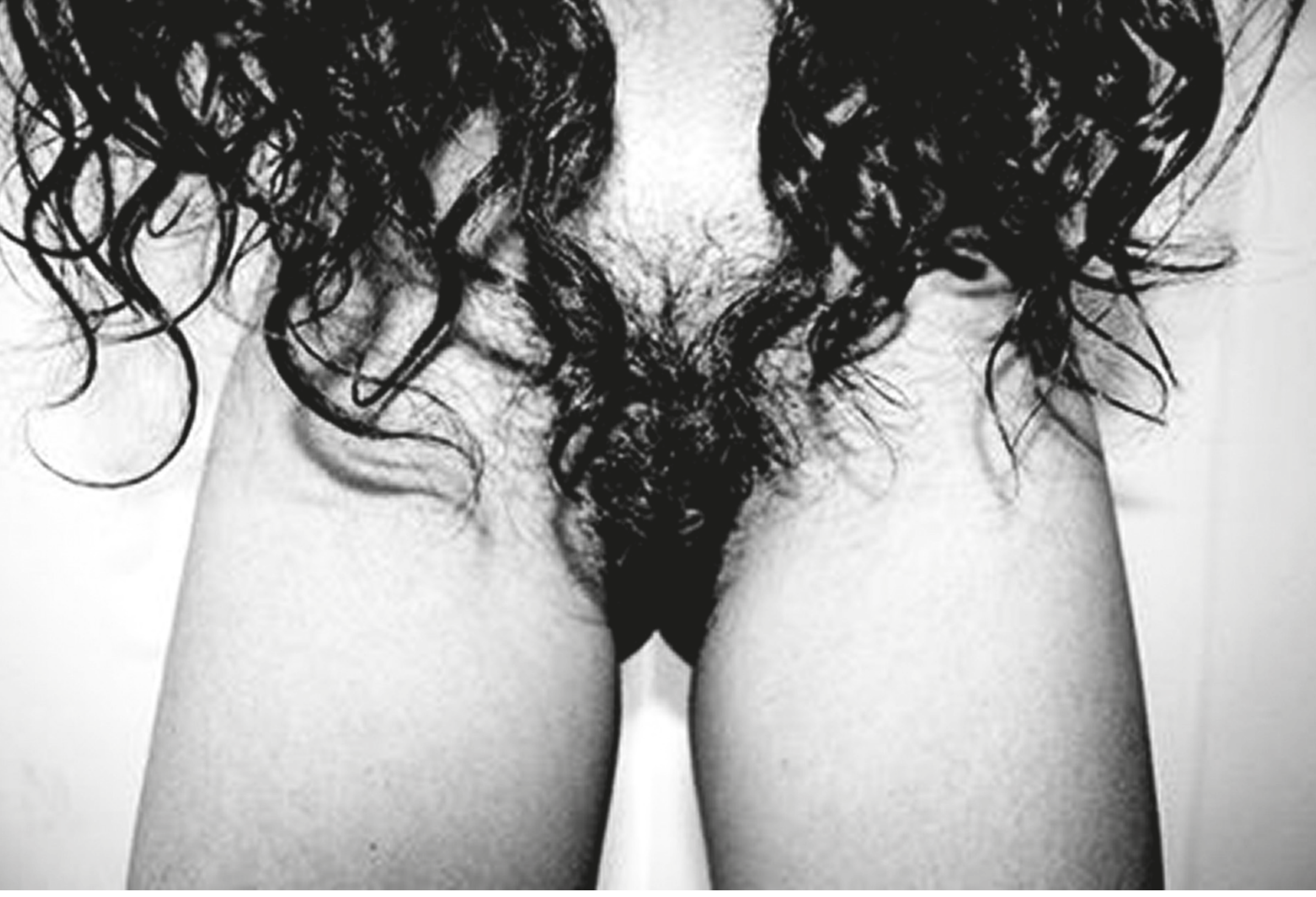

Autorretrato Maru Florecida

Es por eso que es una transnacionalidad que no solo marca la investigación, sino que también marca nuestras biografías personales y de activismo ya que son territorios en los que hemos habitado o estamos habitando, por lo cual son los elegidos para esta investigación. Posteriormente estos datos han sido compartidos y reflexionados por ambas investigadoras dándole una perspectiva transnacional al estudio, y siempre cuidando las diferencias sociopolíticas e históricas de cada uno de ellos.

Como se explicó en el inicio del epígrafe, es importante mencionar que en esta investigación las autoras no solo ejercemos como investigadoras, sino también como feministas y firmes activistas en torno a repensar la corporalidad y la menstruación como forma de re-apropiación del cuerpo de las mujeres, participando en distintas instancias 
abocadas al tema, como talleres, charlas y procesos de autoformación. El hecho de que nuestro activismo se entronque con el trabajo de campo y las entrevistas, ha dado lugar a que algunas informantes, en muchos casos mujeres con quienes tenemos relaciones de cercanía, hayan re-pensado su corporalidad y su vínculo con el ciclo menstrual, gracias a distintos procesos de intercambio de experiencias y significados.

Es importante relevar este hecho, dado que durante el trabajo de campo se ha entrelazado el activismo y la investigación en algunos itinerarios corporales, donde han participado activistas o desde nuestra participación se ha influenciado que las informantes reflexionen en torno al tema de la menstruación. Tomando en cuenta lo anterior, hemos intentado que esta influencia no se refleje en los itinerarios corporales, y de hecho, hay mujeres con una visión crítica de este discurso. No obstante, no deja de ser significativo que como investigadoras en este caso, nos alejamos del mero papel de investigadora "objetiva" para admitir nuestra propia ideología durante el proceso etnográfico. En las conclusiones finales nosotras reflexionamos a través de la conceptualización de nuestros cuerpos, el de las informantes y nos consideramos sujetas activas dentro de este debate, sin separarnos del resto de mujeres solo por el hecho de ser investigadoras. No es el caso, cuando hacemos un resumen de las informaciones que nos han dado las mujeres menstruantes a través de sus itinerarios, que es el siguiente punto, tomando la decisión de no participar como informadoras; respetando el hecho de que no son nuestras historias de vida menstruantes, aunque también hemos sido parte activa en estas historias de vida.

\title{
Itinerarios corporales menstruantes
}

\author{
Todos los días, sin excepción, todxs y cada unx de nosotrxs bailamos en coreografías cotidianas que se nos han \\ naturalizado corporalmente y que trazan los territorios por donde nuestros cuerpos- en definitiva, nuestras identidades \\ en proceso vital - pueden transitar.
}

Meri Torras (2016, p. 4).

A lo largo de estos itinerarios corporales las mujeres nos han hablado de su menstruación sin pudor. Han existido informantes que se han ofrecido hacer los itinerarios mostrando una total libertad y normalidad en expresar cómo es su menstruación y el ciclo que les acompaña. Nos parece importante destacar en primera instancia que esta ausencia de pudor para escribir y hablar sobre un tema estigmatizado a nivel público e histórico no es visualizado en nuestro estudio. Pese a que la menstruación es un tabú en el espacio público, no es así en el espacio privado, donde las mujeres sí desarrollan conversaciones normalizadoras sobre la menstruación, las incomodidades que vivencian en relación con ella, los cambios hormonales que tienen durante el ciclo, entre otros temas. Desde allí, es que esta 
investigación hace emerger al ámbito de lo público las conversaciones más cotidianas que se dan entre mujeres en el ámbito privado y de esta forma llevar al debate abierto una cuestión que ha quedado relegada al mundo íntimo de las mujeres.

De estos itinerarios entregaremos apuntes generales, mostrando pequeños trozos, pero con un gran valor etnográfico para entender a las mujeres y la relación que establecen con su cuerpo. Estos inician con el recuerdo de la primera menstruación y lo difícil que se les hacía entender por qué sucedía esto y los cambios experienciados en su cuerpo. Explicaciones que en el caso de nuestras informantes siempre quedaban relegadas a las mujeres de la familia y que nunca se extendían más allá de la explicación de que el ciclo menstrual dura 28 días y los cuidados que debe tomar la mujer durante la menstruación en cuanto a su higiene.

\footnotetext{
No he sido muy consciente con mi menstruación. Me llegó a los 14 años (creo) y ya está... no me hice muchas preguntas ni nadie me habló mucho de eso. La pareja de mi papá en este entonces me explicó vagamente lo que era y los "cuidados de higiene" mensuales que debía tener. Recuerdo que entre los 17 y 23 años algunas menstruaciones eran sumamente dolorosas (nocturnas), terminando en urgencia. Tras varios exámenes médicos, no se concluyó nada y finalmente solo andaba con unas pastillas específicas por si me venían los dolores.

C. Santiago, Chile.
}

Además de esta falta de explicación y pedagogía, y por tanto, de comprensión del cuerpo y del ciclo menstrual, otra de las inquietudes constantes entre las informantes es el olvido por parte del sistema de salud hegemónico y la sociedad ante los problemas de las mujeres, su menstruación, y el ciclo menstrual. Es por ello que las quejas por las faltas de respuestas concretas para superar molestias durante el ciclo, entre ellos el síndrome premestrual, por parte de la biomedicina, hay que añadirle el tabú que existe para expresar estas molestias tanto en el ámbito laboral como familiar, incluyendo las parejas o amigos masculinos, que no entienden los cambios de comportamiento del ciclo menstrual a causa de los cambios hormonales, ni las molestias que pueden desencadenar en el cuerpo o incluso la estigmatización que rodea a los productos relacionados con la higiene femenina. De esta manera el proceso del ciclo menstrual y su sangrado no ocupan un papel relevante en el sistema salud hegemónico y los ámbitos sociales relacionados con su mundo laboral, familiar y de amistad, espacios compartidos con hombres, pero que en esos mismos espacios la menstruación y todo el proceso cíclico si es compartido, de manera privada, entre las mujeres menstruantes. .

Pero sin duda, la tensión generalizada entre las mujeres menstruantes, sobre todo entre los veinticinco y los cuarenta años, es la excesiva medicalización de la menstruación. Durante el periodo de edad fértil las mujeres toman la píldora anticonceptiva entre diez a quince años de forma continuada. Esto por diversas causas, entre las que 
cuentan que sus primeras menstruaciones fueron dolorosas, con escasas o ausentes explicaciones respecto a que el sangrado del ciclo menstrual pueda llegar con dolores en el útero o bien porque tuvieron menstruaciones fuera de los márgenes "normales" de los 28 días, lo que se resolvió con medicación para cumplir con el ciclo reglamentado, o bien por la aparición de pólipos quísticos a edades tempranas. En otros casos, se les asignaba la píldora como método anticonceptivo cuando comenzaron a tener una vida sexual activa o incluso hay casos que se les recetan estas píldoras por problemas asociados a cuadros de acné que responden positivamente a la regulación hormonal entregada por el medicamento.

\footnotetext{
He tenido controles desde muy joven porque siempre he tenido pólipos y ya desde muy joven empecé a tomar la píldora, como a los 18 años. Incluso con 24 años me tuvieron que operar para extraerme uno de los pólipos y lo recuerdo como muy doloroso, porque el médico me dijo que tendría pólipos siempre y me daría problemas para ser madre. Lloré mucho cuando me dijo esto. Este mismo médico me aconsejó tomar la vacuna contra el papiloma, pero al recibirla, por ser mayor de 24 años la seguridad social no me la cubría y me la tuvo que pagar mi madre.

De todas formas, ni antes, ni después de la operación he tenido dolores menstruales fuertes y la regla siempre me ha llegado cada 28 o 30 días, excepto cuando he dejado de tomar la píldora... En la actualidad, ya tengo 35 años, he decidido dejar de tomarla por cansancio y porque estoy harta de cargarme el método anticonceptivo de las relaciones sexuales de pareja, porque yo soy la que tomo la píldora anticonceptiva, pero al estar dos meses sin tomarla me ha aumentado el nivel de grasa en la piel y cabello por eso estoy entre volverla a tomarla o no.

G. Islas Canarias, España.
}

Observamos que en gran parte de los casos las mujeres ven como única opción consumir la píldora porque es presentada desde el discurso social como opción única, sumado a que en los periodos en los cuales han dejado de tomar la píldora el resultado supone trastornos en su cuerpo, como por ejemplo: aumento de peso, aumento de la retención de líquidos, algunas incluso mencionan sufrir episodios de migrañas que les perjudican en el desarrollo de su vida cotidiana y con ello deciden volver a tomarla. Algunas mujeres han optado por dejarla de forma definitiva, y otras a pesar de la decisión vuelven a consumirlas ante las problemáticas de salud que se les presentan, con el objetivo de "normalizar" sus ciclos hormonales tal cual la píldora les permitía. Las únicas que no han visto cambios en el cuerpo después de dejarlas de tomar son aquellas que pasaron el embarazo y la lactancia y que decidieron no retomar su consumo una vez terminada el periodo de lactancia. En estos casos, ellas hacen la relación que los cambios producidos en su cuerpo a raíz de la maternidad les ayudaron a normalizar lo que consideraban irregularidades en su ciclo menstrual. 
Con todo ello, también surgen itinerarios de mujeres, no mayoritarios y más normalizados dentro del territorio catalán, que en un momento determinado de su vida menstruante hacen una relectura de su menstruación, cambiando la cosmovisión que tienen de esta y de su cuerpo y por tanto de ellas como sujetos individuales y sociales.

En cuanto a mi menstruación nunca he tenido problemas, pero a partir del uso de la copa menstrual fue cuando mi visión sobre la menstruación y mi cuerpo cambió, porque al usar la copa entras en contacto con tu menstruación, con tu sangre, con tus tiempos menstruales y empiezas a conocerte más como mujer y ver que no eres igual a los hombres, que "somos iguales pero con diferentes cuerpos". Esta sociedad se olvida de enseñar a escucharnos a nosotras y a nuestro cuerpo y a nuestros ciclos menstruales, y nos impone ponernos unos pañales (se refiere a las compresas). Vamos al médico y que nos diga lo que nos pasa y así nos olvidamos de escucharnos. Ojo, no quiero decir que no haya que ir hacerse chequeos ginecológicos porque yo me los hago, pero nosotras somos las primeras que conocemos nuestro cuerpo y yo esto lo sentí desde que empecé a usar la copa menstrual.

Además es un producto natural y ecológico (refiriéndose a la copa) y no son productos sintéticos que se introducen dentro de la vagina y luego al tirarlos son contaminantes y por eso es mucho más sano para todos en este mundo.

A. Cataluña, España.

En estos casos se trata de mujeres que usan productos ecológicos para el sangrado (copas menstruales o toallitas de tela) y a través del uso de estos productos surge un interés por conocer y superar los estigmas que existen en la menstruación. En algunos casos explican que ya el mero hecho de relacionarse con la sangre de otra manera, teniendo un contacto directo con ella, devienen a plantearse la menstruación como algo normal y natural para las mujeres, sin la asociación a la enfermedad, como es el discurso dominante. Ellas relacionan la menstruación como una parte integrada a su corporalidad que ha sido anulada del cuerpo de la mujer por parte de los procesos de socialización y que debido a esa anulación la mujer no ha sabido darle lectura, perdiendo compresión respecto al fenómeno de la menstruación y los ciclos menstruales como un proceso natural de sus cuerpos. En estos casos, estas mujeres informantes muestran una crítica o disidencia a los sistemas hegemónicos actuales, algunas se definen así mismas como feministas y anticapitalistas y otras como ecologistas y feministas, pero en todas ellas hay una crítica al trato que recibe su cuerpo por parte del sistema hegemónico y no dejan de incorporar ese discurso de subalternidad a la relación que tienen con su cuerpo menstruante.

Es por ello que teniendo en cuenta que parte de las estrategias colonizadoras de la medicina occidental moderna, y del discurso médico científico, es imponer un modelo universal, único, que gestione diferentes aspectos de la vida humana y cómo estas estrategias se extienden y utilizan a grupos sociales, definidos en función de las clases, el género y la etnia (Jiménez, 2008). Es coherente que estos discursos disidentes y críticos aparezcan con mayor frecuencia y profundidad en mujeres informantes residentes en Cataluña, y más bien difuminados en mujeres residentes en Chile e Islas Canarias, replicando lógicas de poder y subordinación coherentes con elementos de centro/periferia. 


\section{Descolonizando el cuerpo de la mujer}

Viví la menstruación como ajena a mi cuerpo. El único significado que tenía para mí era que me hacía "mujer", aunque tampoco entendía muy bien qué significaba eso, más allá de que mi cuerpo estaba biológicamente preparado para engendrar vida.

J. Santiago, Chile.

En esta investigación-activista, nosotras partimos de que la menstruación y el ciclo menstrual es un proceso invisibilizado por la sociedad, una invisibilización más a la que se ve sometida la mujer y su cuerpo por parte del sistema hegemónico en el cual vivimos, donde el cuerpo de la mujer y sus órganos ha sido colonizado, aculturizado y ocupado por la cultura patriarcal (Valls, 2009).

Es de ahí donde nosotras tomamos el pensamiento decolonial (Jiménez, 2008) como herramienta de reflexión y relectura para repensar un cuerpo que es regulado, controlado, normativizado y discriminado primero por el hecho de ser un cuerpo de mujer, y posteriormente elementos como raza, clase y género se cruzan en la configuración de las relaciones interpersonales, intergrupales y con el mundo. Un cuerpo que es conceptualizado como un cuerpo fallido, ya que no es cuerpo de hombre, el cuerpo de la mujer es considerado como "el no varón" (Valls, 2009, p. 10), lo cual conlleva que la mujer no sienta los órganos y sus funciones como suyos, convirtiéndola en víctima de su propio cuerpo. Un cuerpo que se presenta como una metáfora de carencia, de ausencia; tal vez una excesiva proximidad con la naturaleza, por lo cual se vuelve necesario algún mecanismo de control sobre este.

Un mecanismo de control que se mantiene en la cosmovisión de la sociedad, y por tanto, en nuestra cultura popular, y que es transmitido desde niñas cuando comenzamos a conocer nuestros cuerpos. No en vano, y como explica el itinerario al principio del epígrafe, ya con nuestra primera menstruación se nos explica que con el ciclo menstrual y el sangrado nos convertimos en cuerpos reproductivos, obviando la serie de cambios que ocurrirán en nuestros cuerpos, y el hecho de que el ciclo menstrual es parte de nuestra salud como mujeres y que la menstruación nos acompañarán durante unos cuarenta y cinco años de nuestra vida, aunque no hagamos uso de esa capacidad reproductiva. En la mayor parte de los casos, esta ausencia de explicaciones se debe al desconocimiento del propio ciclo por parte de la sociedad adulta que son los que hacen la transmisión de saberes a las generaciones más jóvenes. De esa falta de explicación y entendimiento de lo que pasa en nuestro cuerpo surge una desconexión total hacía la menstruación a la cual solo le damos una importancia cuando nos dice si estamos o no embarazadas.

Esta desconexión se mantiene y sostiene en la práctica de delegar el cuidado de nuestro cuerpo al sistema de salud hegemónico que, como ya se mencionó anteriormente, es una institución que entrega orden social y por ende se 
rige por los sesgos sociales que representa, manteniendo una visión patriarcal, colonial y mercantilista del cuerpo de la mujer. De esta manera, al medicalizar el cuerpo femenino logra otorgarle un valor productivo y reproductivo e invisibiliza los síntomas propios del ciclo menstrual los cuales son significados por la sociedad y el sistema médico que lo representa, como una pérdida de razón del ser humano, entendiendo el ser humano como el varón, y por tanto, con el mandato de desaparecer de nuestro cuerpo por insanos (Northrup, 1999; Salvia, 2012). Esta crítica hacia la medicalización que muestran las mujeres nos permite observar cómo nuestro cuerpo siguen siendo un desconocido, un invisible y cómo es que este proceso de invisibilización sostiene que nuestro cuerpo siga siendo una fuente de opresión y poder (Harcourt, 2011).

En definitiva, lo expuesto en esta investigación habla sobre cómo no es posible considerar de forma dicotómica las relaciones de poder y subordinación. En el caso de este estudio, se pone en evidencia el control del conocimiento y cómo este afecta a la subjetividad de las mujeres, poniendo además en manifiesto que no es posible hablar de las mujeres como si fueran un solo bloque, sino comenzar a desvelar la construcción diferencial del género desde el sistema moderno/colonial, lo cual permitiría la emergencia de otros discursos desde distintas perspectivas y experiencias que compartan la voluntad opositora hacia lo hegemónico.

Por ello, es necesario que en los estudios feministas se trabaje en una producción teórica más contextual. No es posible desconocer que la academia sigue siendo occidental y masculina y es desde donde emerge el conocimiento y la teoría, lo cual perpetúa la relación centro-periferia, tomando en este caso la dicotomía centro-periferia como la de varón-mujer, y en la que los territorios subalternos aportan solamente como campo de aplicación de los conceptos y relatos de las experiencias. Tampoco se trata de invisibilizar el aporte que los movimientos feministas han producido, sino de problematizar que las propuestas del ámbito del feminismo, en su mayor parte para ser consideradas oficialmente, deben cumplir la condición de compartir los mismos presupuestos teóricos coherentes con parámetros occidentales, modernos y coloniales, y por tanto, se configuran solo como un campo de ampliación del mismo sistema de pensamiento (Quiroga, 2011).

Es por esto, que creemos por relevancia enfocar la investigación y la praxis en temas como la menstruación y los ciclos menstruales. Dado que estos temas nos permite abrir un espacio para la construcción de un nuevo imaginario que se nutra de una novedosa intersubjetividad desde la reinvención del cuerpo, la sexualidad, la identidad femenina y los espacios imaginarios de pertenencia, además de aprehender las particularidades de las luchas de las mujeres sin imponerles los límites de las ciencias sociales universitarias academicistas. Hablamos de corporalidad porque es el lugar desde donde nosotras hablamos, sentimos y vemos el mundo. No debemos olvidar que el cuerpo es un lugar de discriminación, pero también de resistencia y contestación, por tanto el empoderamiento de nuestro cuerpo va unido al empoderamiento femenino y social (Harcourt, 2011; Esteban, 2004). 


\section{Referencias}

Berbel, S., Pi-Sunyer, M.T. (2001). El cuerpo silenciado: una aproximación a la identidad femenina. Barcelona: Viena Edicions.

Corvalán, A. (2013). Intervención en torno al autoconocimiento del ciclo menstrual: mi cuerpo habla. Barcelona: Tesis de máster de la Universitat de Barcelona.

Esteban, M.L. (2004). Antropología del cuerpo, género, itinerarios corporales, identidad y cambio. Barcelona: Ediciones Bellaterra.

Esteban, M.L. (2008). Etnografía, itinerarios corporales y cambio social: apuntes teóricos y metodológicos. En Elixabete Imaz (comp.). La materialidad de la identidad. Donostia-San Sebastián: Hariadna Editoriala.

Esteban, M.L. (2011). Crítica del pensamiento amoroso. Barcelona: Ediciones Bellaterra.

Fabiónavá, D. (2009). La luna en ti. (Archivo en video). Madrid: Ubak Producciones.

Guilló, M. (2013). La in-corporación de la investigación política de la menstruación y cuerpos reproductivos. Revista Nómada (39). Bogotá

Harcourt, W. (2011). Desarrollo y políticas corporales. Debates críticos en género y desarrollo. Barcelona: Ediciones Bellaterra.

Houppert, K. (2000). La menstruación: desmontando el último tabú femenino. Barcelona: Editorial Juventud.

Jiménez, I. (2008). Género, sanidad y colonialidad. En Mignolo, W. (comp.). Género y descolonialidad: hacia un feminismo decolonial. Buenos Aires: Ediciones del Signo.

Lugones, M. (2008). Colonialidad y Género. Tabula Rasa, (9), 73-102. Recuperado el 22 de october de 2017, de http://www.scielo. org.co/scielo.php?script=sci_arttext\&pid=S1794-24892008000200006\&lng=en\&tlng=es.

Mignolo, W. (2007). Walter Mignolo: una vida dedicada al proyecto decolonial. Revista Nómadas, (26).

Northrup, C. (1999). Cuerpo de mujer, sabiduría de mujer: Una guía para la salud física y emocional. Barcelona: Ediciones Urano, S.A.

Perona, A.J. (1995). La construcción del concepto de ciudadanía en la modernidad. Revista Arena: Revista de Historia de las Mujeres, (2).

Quiroga, N. (2011). Economía del cuidado. Reflexiones para un feminismo decolonial. Revista Casa de la Mujer, 20. Costa Rica.

Salvia, A. (2012). Viaje al ciclo menstrual. Barcelona: Licencia Creative Commons.

Torras, M. (2016). Lo que puede el baile: escrituras de los cuerpos en movimiento y vínculo. En Gutiérrez M. A. (comp.). Entre-Di-

chos-Cuerpos. Coreografías de los géneros y la sexualidad. Buenos Aires: Ediciones Godot.

Valls, C. (2006). La menstruación: de la invisibilidad a la abolición. Duoda: Revista d'estudis feministes, 31.

Valls, C. (2009). Mujeres, salud y poder. Barcelona: Cátedra.

Wolf, N. (2013). Vagina. Una nueva biografía de la sexualidad femenina. Barcelona: Editorial Kiarós. 